\title{
Savoirs techniques, et migrations : l'histoire face aux mythes
}

Useful knowledge, identities and migrations : history faces mythologies

\author{
Liliane Pérez
}

\section{CpenEdition}

\section{Journals}

Édition électronique

URL : http://journals.openedition.org/dht/928

ISSN : $1775-4194$

Éditeur:

Centre d'histoire des techniques et de l'environnement du Cnam (CDHTE-Cnam), Société des élèves du CDHTE-Cnam

\section{Édition imprimée}

Date de publication : 1 juin 2008

Pagination : 3-9

ISBN : 978-2-95-30779-1-9

ISSN : 0417-8726

Référence électronique

Liliane Pérez, «Savoirs techniques, et migrations : I'histoire face aux mythes », Documents pour

I'histoire des techniques [En ligne], 15 I $7^{\text {er }}$ semestre 2008, mis en ligne le 21 octobre 2010, consulté le 07 septembre 2020. URL : http://journals.openedition.org/dht/928 


\title{
Savoirs techniques,
}

\section{identités et migrations: \\ l'histoire face aux mythes}

\section{Liliane Pérez CDHTE-Cnam}

\begin{abstract}
RÉsumé
L'histoire de la transmission des savoirs techniques a largement bénéficié de la mise en cause du modèle diffusionniste qui sous-tendait la notion de transfert technique. Dans cette perspective, les questionnements de méthode ont été déterminants, notamment l'identification du rôle des intermédiaires, individus, groupes, réseaux, essentiels sur le long terme tant que les connaissances restent peu codifiées. L'analyse critique des récits tendant à magnifier le rôle de certains passeurs ou à assigner des compétences techniques à un groupe, qui plus est à une minorité, a été au cœur de la journée d'étude organisée sur le thème " Minorités et circulations techniques ». Les contributeurs, par des études de cas précises, révèlent d'une part l'existence de processus de transmission diffus, dont les acteurs furent souvent modestes ou anonymes et les mobiles complexes à démêler, et proposent d'autre part des pistes de réflexion pour comprendre la place des savoirs techniques dans l'élaboration des constructions identitaires.
\end{abstract}

Résumés et mots clés en anglais sont regroupés en fin de volume, accompagnés des mots clés français.

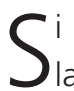
le terme de «savoir » a longtemps été réservé à a connaissance abstraite, et en particulier scientifique, on convient de nos jours de la spécificité et de l'autonomie des savoirs techniques, sans lesquels l'élaboration théorique elle-même ne saurait exister' ${ }^{1}$. Aux côtés des savoirs analytiques, existent des savoirs d'action, fondés sur l'aptitude au projet et sur l'abstraction à partir du réel, par les facultés d'analogie et de synthèse ${ }^{2}$. La reconnaissance épistémologique des

Cet article doit beaucoup à la lecture attentive de Patrice Bret, d'Eugénie Briot et de Catherine Verna. Qu'ils en soient vivement remerciés.

1 Les historiens des sciences ont montré la part déterminante des pratiques, de l'expérimentation, des manipulations et des constructions d'appareils et d'instruments dans l'élaboration des théories scientifiques. Pour des synthèses rappelant l'impact de cette approche sur I'histoire des savoirs techniques, voir Layton E. T., "Technology as knowledge», Technology and culture, XV (1974), pp. 31-41 ; Laudan R., « Natural alliance or forced marriage? Changing relations between the histories of science and technology », Technology and culture, XXXVI, suppl., 1995, S17-S2. 2 Vérin $\mathrm{H}_{\text {., }}$ La gloire des ingénieurs. L'Intelligence technique $d u$ sciences de l'artificiel, dont l'histoire appartient à celle de la technologie comme science de la technique ${ }^{3}$, s'est accompagnée de nombreuses études sur les mo-

XVe au XVIII siècle, Paris, Albin Michel, 1993. Sur le savoir et le geste, dans la lignée de l'anthropologie des techniques, voir : Sigaut F., "Le savoir des couteaux», dans D'Onofrio S. et Joulian F. éd., Dire le savoir-faire. Gestes, techniques et objets, L'Herne. Cahiers d'anthropologie sociale, 2006, pp. 133-139; Archambault de Beaune S., Pour une archéologie du geste, Paris, CNRS Éditions, 2000

3 Voir le dossier sur la technologie paru dans les Documents pour I'histoire des techniques, nouv. série, n 14, 2007 : Guillerme J., Sebestik J., "Les commencements de la technologie » (reprint de Thalès, 1968, XII, pp.1-72), pp. 49-122 ; Sebestik J., « ibid. Postface/Préface ? », pp.123-133 ; Vérin H., " La technologie: science autonome ou science intermédiaire? », pp. 134-143. Voir également : Le Moigne J.-L. et Vérin H., « Sur le processus d'autonomisation des sciences du génie », dans De la technique à la technologie, Cahiers science-technologie-société, Paris, 1984, pp. 42-55 ; Sigaut F., "Haudricourt et la technologie », dans Haudricourt A.-G., La Technologie science humaine. Recherches d'histoire et d'ethnologie des techniques, Paris, Éditions de la MSH, 1987, pp. 9-36. 
dalités particulières d'acquisition, de transmission et d'appropriation des savoirs pratiques.

Loin de se limiter aux institutions pédagogiques et culturelles, l'espace public de la technique est aussi celui de l'entreprise, de la production et de l'échange marchand. Les savoirs techniques vivent au rythme des économies, de l'essor, de l'intégration ou de la contraction des marchés. Leur histoire a partie liée avec I'histoire économique, y compris avec ses concepts. Ainsi, le circuit, clé de voûte de la pensée économique sur la longue durée, a longtemps servi de socle à l'analyse de la diffusion des savoirs techniques en termes de circulation et de transfert. Cependant, si la transmission des savoirs techniques est indissociable des mobilités humaines et du commerce des produits et des équipements, la notion de circulation présente aussi des limites pour la compréhension des processus en jeu. Les historiens des techniques et de l'économie tendent ainsi à prendre leur distance avec les métaphores du circuit, du moins à redéfinir leur emploi ${ }^{4}$. Ce fut le point de départ de la journée d'étude dont nous publions ici les actes. Deux thèmes ont orienté les réflexions.

L'un concerne la mise en cause du modèle diffusionniste, de l'immédiateté des transferts, au profit d'une approche attentive à leur complexité, aux synergies de moyens, techniques et non techniques et qui, lorsqu'ils font défaut, peuvent expliquer les freins et les échecs, l'impossible transmission, les savoirs perdus ${ }^{5}$. L'autre

4 Les articles présentés dans ce recueil sont issus de la journée d'étude «Circulations techniques et minorités » tenue au Conservatoire national des arts et métiers, le 4 juin 2004. Comme tels, ils sont le fruit d'une double entreprise, le séminaire sur les circulations de savoirs techniques et le projet franco-anglais « Culture of commerce and invention in modern Europe ». Le séminaire " Techniques : circulations des hommes, des savoirs et des produits du Moyen Âge à l'époque contemporaine (Orient, Occident) » (2002-2004) a été organisé par Patrice Bret, Robert Carvais, Liliane Pérez, Catherine Verna, dans le cadre d'une collaboration entre le CDHTE-Cnam, I'Université Paris 8 et le Centre Alexandre Koyré-Centre de recherche en histoire des sciences et des techniques (CAK-CRHST). La journée « Circulations techniques et minorités » a été organisée par Patrice Bret, Liliane Pérez et Catherine Verna, avec le concours du CDHTE-Cnam, de I'Université Paris 8, du CAK-CRHST et du Leverhulme Trust Foundation. Le projet financé par le Leverhulme Trust Foundation était dirigé par Maxine Berg (Université de Warwick), en collaboration avec Liliane Pérez pour le CDHTE (2003-2006). La journée d'étude d'Oxford, «A commerce with strangers : trade and technology between East and West », en juin 2004, a utilement complété les travaux de celle du Cnam.

5 Mathias P., « Skills and the diffusion of innovations from Britain in the eighteenth century », Transactions of the royal historical question a trait au poids des appropriations et des usages $^{6}$. Si le concept est classique en histoire culturelle, s'il a largement bénéficié des apports de l'anthropologie de l'altérité7, il a plus lentement cheminé en histoire des techniques tant l'idéologie du progrès, souvent occidental, et son pendant, le paradigme du retard et du rattrapage ont durablement guidé les analyses ${ }^{8}$. De rares auteurs dans le passé, tels Paul Bairoch et Nathan Rosenberg, avaient souligné que les techniques ne circulent pas sans être interprétées, adaptées et que les passeurs ne sont pas de simples relais ${ }^{9}$. Ils remettaient ainsi en cause une vision homogénéisatrice des transferts au profit d'une perception territorialisée des savoirs techniques, d'une prise en compte de la diversité des rythmes de circulation et de la qualité différenciée des espaces de réception ${ }^{10}$.

society, vol. 25, 1977, pp. 93-113 ; Belfanti C. M., « Guilds, patents, and the circulation of technical knowledge: northern Italy during the early modern age », Technology and culture, vol. 45, 2004, pp. 569-589 ; Mokyr J., The gifts of Athena. Historical origins of the knowledge economy, Princeton, Princeton University Press, 2002.

6 Hilaire-Pérez L. , Verna C., « Les circulations techniques : hommes, produits, savoirs au Moyen Âge et à l'époque moderne (Orient, Occident) », dans Cotte M. éd., Les Circulations techniques. En amont de l'innovation : hommes, objets et idées en mouvement, Belfort/Besançon, UTBM/Presses universitaires franc-comtoises, 2004, pp. 11-36; id., «Dissemination of technical knowledge in the middle ages and the early modern history. New approaches and methodological issues », Technology and culture, vol. 47, juillet 2006, pp. 536-565.

7 Gruzinski S., La Pensée métisse, Paris, Fayard, 1999 ; Amselle J.-L., Branchements. Anthropologie de I'universalité des cultures, Paris, Flammarion, 2001

8 L'un des lieux de recherche en ce sens a été le séminaire mené par Yves Cohen et Kapil Raj à l'École des hautes études en sciences sociales, "Savoirs, pratiques et objets : localités et circulations » où nous avions présenté les résultats du séminaire en 2005. Ces problématiques furent aussi au cœur de l'ACI-CNRS Histoire des savoirs (2003-2007) dirigée par Karine Chemla et dans ce cadre, elles ont structuré la réalisation du lexique du travail de rivière et des activités préparatoires au tannage ; voir Hilaire-Pérez L. et Mille M., « Histoire des savoirs techniques : constitution de corpus de sources et de lexiques du Moyen Âge à l'époque contemporaine » dans Chemla K. et Delbraccio M. éd., Action concertée Histoire des savoirs 2003-2007, Paris, CNRS, 2007, pp. 239-250.

9 Bairoch P., Révolution industrielle et sous-développement, 1963, rééd. Paris-La Haye, Mouton, 1974 ; Rosenberg N., « Economic development and the transfer of technology : some historical perspectives », Technology and culture, vol. 11 1970, pp. 550-575

10 On trouve actuellement des échos à cette problématique dans les débats sur la définition des enjeux et des méthodes de 


\section{Les circulations techniques : enjeux méthodologiques}

Cette approche, attentive à la complexité des processus de transmission, a rencontré peu d'écho immédiat, tant elle suscitait de questions de méthode. L'un des problèmes a trait à l'identification de circuits de diffusion composites, non linéaires, au gré des adaptations et des transpositions de techniques, au fil des oublis et des résurgences de savoirs ${ }^{11}$. Si les appellations géographiques de produits et de procédés ne peuvent être tenues comme l'expression d'une origine réelle ${ }^{12}$, les autres marqueurs et supports de circulation ne sont pas toujours plus fiables. Pendant longtemps, le traité technique a peu servi la transmission des savoirs; d'autres écrits, non techniques tels ceux des notaires, se révèlent parfois plus utiles ${ }^{13}$. Mais bien souvent, les produits et les hommes, individus, groupes, réseaux, ont été les principaux vecteurs (bien que jamais suffisants ${ }^{14}$ ).

Ce thème des intermédiaires culturels a suscité nombre d'études visant à retracer les itinéraires individuels (ouvriers, entrepreneurs, ingénieurs, voyageurs) et les migrations de groupes dans la diffusion des savoirs

I'histoire globale comme world history : voir ainsi Douki C., Minard Ph., «Introduction », dans Histoire globale, histoire connectées : un changement d'échelle historiographique, Bulletin de la Société d'histoire moderne et contemporaine, Revue d'histoire moderne et contemporaine, vol. 54-4 bis, supplément 2007, pp. 7-21. Dans le volume, voir par exemple l'approche critique de Zuniga J.-P., « L'histoire impériale à I'heure de I' "histoire globale". Une perspective atlantique », pp. 54-68.

11 Bret $P$., "La Méditerranée médiatrice des techniques : regards et transferts croisés durant l'expédition d'Égypte (1798-1801) », dans Bourguet M.-N., Nordman D., Panayatopoulos V., Sinarellis M. éd., Enquêtes en Méditerranée. Les expéditions françaises d'Égypte, de Morée et d'Algérie, Athènes, Institut de Recherches Néohelléniques/FNRS, 1999, pp. 79-101.

12 Voir le cas récemment étudié des cosmétiques : Lanoë C., La Poudre et le fard. Une histoire des cosmétiques de la Renaissance aux Lumières, Seyssel, Champ Vallon, 2008 (en particulier le chapitre I pour les taxinomies, telles que «blanc d'Espagne », " poudre de Chypre », " eau de la reine de Hongrie »).

13 Verna $C$., "The notary as a witness: techniques and the dissemination of tacit knowledge ( $\mathrm{XIV}^{\text {th }}-\mathrm{XV}^{\text {th }}$ centuries) », dans Córdoba de la Llave R. éd., Craft treatises and handbooks: the dissemination of technical knowledge in the Middle Ages, sous presse.

14 Cotte M., De l'espionnage industriel à la veille technologique, Belfort/Besançon, UTBM/Presses universitaires de France-Comté, 2005 ; Dolza L., " How did they know ? The art of dyeing in late eighteenth-century Piedmont », dans Fox R. et Nieto-Galan A., Natural dyestuffs and industry in Europe, 1750-1880, Canton (Mass.), Watson, 1999, pp. 129-160. techniques. Cependant, à nouveau, ces approches ont montré leurs limites, soit qu'elles tendent à magnifier le rôle de certains passeurs, soit qu'elles assignent des compétences techniques spécifiques à un groupe national, ethnique, religieux, socioculturel, qui plus est à une minorité. L'analyse micro-historique des réseaux, des phénomènes de ségrégation et d'intégration, révèle au contraire des processus de transmission diffus, dont les acteurs sont souvent modestes ou anonymes et les mobiles complexes à démêler ${ }^{15}$. Au cœur des questions de méthode se pose donc celle des enjeux épistémologiques de l'étude des migrations, en particulier des déplacements de groupes et de communautés, tant les constructions idéologiques ont été puissantes dans ce domaine ${ }^{16}$.

Si les recherches sur la mobilité du capital humain ont beaucoup progressé, si I'on comprend de plus en plus finement les différents modèles migratoires dans le monde du travail sur la longue durée, migrations individuelles ou collectives, circulaires, en chaîne ou opportunistes, déplacements forcés, suscités ou volontaires, temporaires, saisonniers ou pérennes, au gré de la gestion municipale ou étatique des étrangers, I'analyse des minorités pose des problèmes particuliers qui dépassent le seul repérage des circuits, des réseaux et des statuts de migrants, des modes de transmission et des facteurs d'émigration ou d'attraction ${ }^{17}$. Elle introduit

15 Mitchell D. éd., Goldsmiths, silversmiths, and bankers: innovation and the transfer of skill, 1550-1750, Stroud, Sutton Publishing, 1995

16 Ciriacono S., « Migration, minorities, and technology transfer in early modern Europe », The journal of European economic history, vol. 34, printemps 2005, pp. 43-64.

17 Epstein S. R., " Journeymen, mobility, and the circulation of technical knowledge », dans Hilaire-Pérez L., Garçon A.-F. éd., Les Chemins de la nouveauté. Inventer, innover au regard de I'histoire, Paris, CTHS, 2004, pp.411-430 ; id., " Crafts, guilds, apprenticeship, and technological change in pre-industrial Europe », Journal of economic history, vol. 58, 1998, pp. 684-713 ; Pinto A., Verna C., «Les Basques dans les forges de Catalogne : migration, culture technique et industrie rurale ( $x v^{e}-x v l^{e}$ siècles) ", dans Guérassimoff E., Maitte C., Mandé I., Martini M., Terrier D. éd., Migrations et cultures d'entreprise : diversité et échanges dans le monde, $x V^{e}$ - $x x^{e}$ siècle, Valenciennes, Presses universitaires de Valenciennes-Les Belles Lettres, à paraître ; Maitte $C$., « Corporation et politique au village: Altare entre migrations et processus de différenciation sociale ", Revue historique, vol. 617, 2001, pp. 45-79 ; Dolza L. et Maitte C., " "L'appel aux étrangers". Circulations et intégration des savoir-faire dans le Piémont de l'Ancien Régime : le cas de la teinture en laine et de la verrerie », dans Cotte M. éd., Les Circulations techniques, op. cit., pp. 77-94 ; Maitte C. et Martini M. éd., « Pratiques migra- 
une dimension identitaire, un lien entre savoirs techniques et appartenances de groupe, qui impose une mise à distance de l'historiographie ainsi que des discours des contemporains, tant les récits qui assignent des techniques à une origine communautaire, ethnique ont été des vecteurs de revendications politiques ou nationalistes et ont participé de la construction de récits mythiques que les historiens, maintenant, s'attachent à comprendre ${ }^{18}$.

\section{Groupes, réseaux, minorités}

L'historiographie des minorités et des transferts techniques est marquée par les travaux de Warren C. Scoville sur les huguenots ${ }^{19}$, dans la lignée des thèses de Max Weber et de Werner Sombart liant judaïsme et capitalisme, auxquelles Scoville fait explicitement référence. Le contexte des années 1950 est cependant différent et Scoville inscrit aussi son propos dans l'actualité des migrations et des transferts nés des guerres et de la colonisation. Ce rapport au présent explique les grandes lignes de sa pensée. Sa problématique est celle de l'avance et du retard technique, plus que l'analyse anthropologique des liens entre religion et économie, minorités et savoir-faire. La construction identitaire, dans ses rapports à la praxis, n'est pas questionnée par Scoville, contrairement à Sombart, à Weber, ou à Marx. Loin de toute approche anthropologique liant une pratique économique ou une technique à sa dimension sociale et symbolique, Scoville tente de mesurer I'impact des migrants persécutés sur les économies des pays d'accueil au fil d'une énumération des vagues migratoires et des spécialités introduites par les réfugiés. Le lien entre savoirs professionnels et identités de groupe n'est pas questionné. C'est une histoire des flux et de

toires et cultures d'entreprise dans la longue durée », Migrations et Sociétés, décembre 2006, vol. 18, n¹08.

18 Cantelaube J., La Forge à la Catalane dans les Pyrénées ariégeoises: une industrie à la montagne, $X V I^{e}-x I x^{e}$ siècles, Toulouse, collection Méridiennes, série Histoire \& Techniques, 2005 ; Verna C., "Forges catalanes : la question des origines », dans Zimmermann M. éd., Le Moyen Âge dans les Pyrénées catalanes. Art, culture et société, Études roussillonnaises. Revue d'histoire et d'archéologie méditerranéennes, t. 21, 2005, pp. 55-62. 19 Scoville W. C., «The Huguenots and the diffusion of technology », Journal of political economy, vol. 6, 1952, pp. 294-311, 392-411; id., " Minority migrations and the diffusion of technology ", Journal of economic history, vol. 11, 1951, pp.347-360; id., The persecution of Huguenots and French economic development, 1680-1720, Berkeley, California University Press, 1960. leur impact, immergée dans l'actualité des transferts de connaissances et des mouvements de populations du milieu $d u x x^{e}$ siècle. Non seulement les pays occidentaux se posent alors en modèles de croissance et en pourvoyeurs de solutions techniques, mais l'émigration des juifs d'Europe aux États-Unis et surtout au ProcheOrient fournit un exemple immédiat de déplacement de minorités et Scoville tente explicitement la comparaison avec les huguenots ${ }^{20}$. Le modèle historique, révélant le rôle des mobilités dans les transmissions de savoir-faire notamment entre pays ne maîtrisant pas les mêmes techniques, doit servir de grille pour interroger l'impact des exodes contemporains sur les économies d'accueil, selon leur degré de développement. Le modèle diffusionniste, alors en plein essor, érige les mobilités comme facteur déterminant des circulations de savoirs dans les économies passées ${ }^{21}$.

Néanmoins, les catégories forgées par Scoville pour mener l'analyse des flux migratoires ont conservé un intérêt. L'auteur distingue ainsi les groupes et les minorités. Les groupes d'individus sont engagés dans un même métier ou des métiers connexes, comme les artisans débauchés par Colbert que citait Scoville, ou ensuite ceux recrutés par John Law et étudiés par John R. Harris, une centaine, appartenant aux métiers du bois et du métal, le plus souvent horlogers ${ }^{22}$. Ces migrations des groupes professionnels sont de mieux en mieux connues ; elles ont été analysées sur le plan des politiques migratoires, des recrutements et des filières (hommes, informations, matériels) entre centres de départ et centres d'accueil. Pour autant, la question des savoirs reste entière, de même que le fonctionnement des équipes (les hiérarchies, les langues). L'article d'Éric Reppel dans le présent volume, sur les armuriers lombards, allemands et flamands établis à Tours au xvl ${ }^{\mathrm{e}}$ siècle en fournit un bon exemple.

À la différence des groupes, selon la distinction de Scoville, les minorités constituent des populations plus nombreuses, et surtout isolées, repoussées ou expulsées par des barrières religieuses, politiques, culturelles ou sociales. Elles sont au cœur des processus de construc-

20 Scoville W. C., "Minority migrations and the diffusion of technology », op. cit., pp. 359-360.

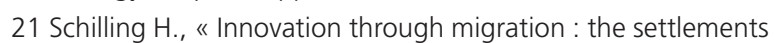
of Calvinistic Netherlanders in sixteenth and seventeenth century central and western Europe », Social history, vol. 16, 1983, pp. 7-33.

22 Harris J. R., Industrial espionage and technology transfer. Britain and France in the 18th-century, Aldershot, Ashgate, 1998. 
tion des États. Elles-mêmes forment des micro-sociétés, avec leurs règles, leurs institutions, leurs « modes de vie », leurs activités et leurs savoirs, en rien réductibles à un unique profil professionnel. Les minorités ne migrent pas en tant que «craft groups ». Leur organisation interne et le fait communautaire priment sur les seules activités, elles-mêmes diverses. Cette dimension culturelle, politique et identitaire explique, selon Scoville, l'impact direct et immédiat ( " pervasive and rapid effect ») des migrations de minorités sur les économies d'accueil, plus fort que celui des simples groupes professionnels et a fortiori, des individus ${ }^{23}$. Dans la gradation établie par l'auteur entre transmission diffuse, « par radiation », et accélérée, « par migration », les minorités sont les vecteurs des transformations techniques et économiques les plus marquantes. L'auteur associait ces transferts à des innovations techniques, radicales, en rien assimilables au processus d'imitation diffus, « le plus normal » des modes de transmission selon lui. Les minorités, parce qu'elles affrontent l'adversité des ruptures avec les pays d'origine, les persécutions et les incertitudes, et parce qu'elles font corps, possèdent la hardiesse d'entreprendre (" hardihood to venture ») essentielle à toute innovation. Chez Scoville, un même héroïsme unit les migrants et les hommes de progrès.

Cette approche n'est plus de mise tant les processus de transmission apparaissent complexes, quels qu'ils soient. En l'espace d'une génération, les perspectives ont évolué, à la fois dans la perception des transferts techniques, moins immédiats et systématiques qu'on ne I'avait pensé, et dans l'analyse des identités culturelles, en rien réductibles, assignables à des savoirs techniques particuliers ou à identités professionnelles ${ }^{24}$. La matu-

23 Scoville W. C., "Minority migrations and the diffusion of technology », op. cit., cp. 351.

24 Pour cette problématique dans le monde contemporain, voir Green N. L., Du Sentier à la Septième Avenue. La confection et les immigrés, Paris-New York, 1880-1980, Paris, Le Seuil, 1998. Pour des époques antérieures, on se reportera utilement à la remise en cause des stéréotypes assignant des techniques aux musulmans sans prendre en compte la diversité des minorités musulmanes, en rien réductible à une minorité en tant que telle, puisque pour certains c'est la Reconquête qui fait d'eux une minorité, pour d'autres c'est l'esclavage, comme le révèlent les fouilles récentes d'un atelier céramique près de Marseille : voir Marchesi $\mathrm{H}_{\text {., }}$ Thiriot J. et Vallauri L. éd., Marseille, les ateliers de potiers du xIIr siècle et le quartier Sainte-Barbe ( $V^{e}-x V^{e}$ S.), Paris, MSH, 1997. Sur les relations entre chrétiens et musulmans, et entre Orient et Occident, voir Lorhmann D., "Échanges techniques entre Orient et Occident au temps des Croisades », dans Draelants I., Tihon A. et Van Den Abeele B. éd., Occident et Proche-Orient. Contacts ration des historiens des techniques sur le sujet, liées aux nouvelles convergences avec l'anthropologie ${ }^{25}$, s'est accompagnée d'un élargissement des champs d'étude, d'une diversification, dans le temps et dans l'espace, dont témoignent pleinement les travaux présentés dans ce recueil.

Les travaux récents sur les huguenots revoient ainsi à la baisse leur rôle dans la transmission des savoirs techniques. Des mythes tombent, comme l'origine huguenote de la coutellerie de Sheffield mise en cause par David Hey ${ }^{26}$. De même, les études menées dans I'orfèvrerie révèlent l'impact diffus des réfugiés, à l'échelle d'une communauté dont l'intégration s'est faite de manière silencieuse, par les réseaux de sous-traitance et par la circulation de patrons et de modèles, dans de nombreux métiers, de manière transverse, tant les continentaux excellent dans le dessin, la façon et la conception des formes ${ }^{27}$. À nouveau se pose la question de l'identification des individus, des ouvriers silencieux et souvent illégaux, mal acceptés par les corporations, absents des inventaires listant les biens qu'ils ont pourtant produits et fournis aux fabricants natifs ${ }^{28}$.

Des pistes existent cependant. Les travaux en cours invitent à se tourner vers le marché du travail. David

scientifiques au temps des croisades, Turnhout, Brepols, 2000, pp. 117-144

25 Minard Ph., « Histoire et anthropologie, nouvelles convergences ? ", Bulletin de la Société d'histoire moderne et contemporaine, Revue d'histoire moderne et contemporaine, vol. 49-4bis, supplément 2002, pp. 81-84.

26 Hey D., "The origins and early growth of the Hallamshire cutlery and allied trades », dans Chartres J. L., Hey D. éd., English rural society, 1500-1800. Essays in honour of Joan Thirsk, Cambridge, Cambridge University Press, 1990, pp. 343-368.

27 Clifford $H_{\text {., }}$ " The myth of the maker: manufacturing networks in the London goldsmiths trade, 1750-1790 », dans Quickenden K., Quickenden N. A. éd., Silver and jewelry. Production and consumption since 1750, Birmingham, 1995, pp. 5-12 ; id., " "The King's Arms and Feathers": a case study exploring the networks of manufacture operating in the London goldsmiths' trade in the eighteenth century », dans Mitchell D., Goldsmiths, silversmiths, and bankers, op. cit., pp.84-95; id., "In defence of the toyshop: the intriguing case of George Willdey and the Huguenots ", Proceedings of the Huguenot society, vol.27, 1999, pp. 171-188; Hartop C., " Art and industry in 18th-century London: English silver, 1680-1760, from the Alan and Simone Hartman Collection », Proceedings of the Huguenot society, vol. 27, 1998, pp. 50-63.

28 Packer E., « The interaction between English and Huguenot goldsmiths in the late seventeenth and early eighteenth centuries ", dans Mitchell D. éd., Goldsmiths, silversmiths, and bankers, op. cit., pp. 84-95. 
Mitchell, pour le XVIII siècle, montre la diversité des modes transmission via l'apprentissage ${ }^{29}$ : compagnons huguenots placés chez des fabricants natifs, huguenots employés en même temps que des étrangers dans un atelier et travaillant à la confection des mêmes articles, natifs placés en apprentissage chez des huguenots ${ }^{30}$. Un dernier cas existe encore : la diffusion des techniques restreinte à la seule communauté migrante, comme le décrit Lien Luu à Londres au XvI ${ }^{\mathrm{e}}$ siècle ${ }^{31}$. Dans son article pour le présent recueil, elle rappelle que l'installation de minorités étrangères dans des enclaves, spatiales et juridiques, comme la soierie à Bishopgate pour les protestants francophones, favorise la divulgation des techniques dans la communauté, y compris auprès des nouveaux arrivants à qui les membres de la filière d'accueil apprennent le tissage des soieries, quel que soit leur métier d'origine. Face à cette solidarité entre générations de migrants, si ce n'est à ce repli, la transmission aux natifs se fait par une politique volontariste de placement chez les étrangers à qui l'État fixe un nombre maximal d'employés de leur pays.

\section{Savoirs techniques et constructions identitaires : des relectures critiques}

La question de l'identification des savoirs transmis par les communautés migrantes rejoint ainsi la thématique identitaire et ses liens avec la gestion politique des mobilités. Si I'héroïsation des minorités actives n'est plus de mise, les interrogations demeurent sur la spécificité des outsiders, sur la perception par les contemporains de leur rôle économique, de leurs compétences, sur le rôle du Politique dans les représentations qui leur sont attachées ${ }^{32}$. Ce renouveau d'intérêt n'est en rien fortuit, il fait écho au présent, tant « la légitimité sociale et politique se fonde aujourd'hui sur un pouvoir

29 Mitchell D., "Innovation and the transfer of skill in the goldsmiths' trade in Restoration London », dans Mitchell D. éd., Goldsmiths, silversmiths, and bankers, op. cit., pp. 5-22.

30 On retrouve le même motif, le placement de natifs chez des étrangers, dans le cas des protestants allemands du faubourg Saint-Antoine: Thillay A., Le Faubourg Saint-Antoine et ses " faux ouvriers ». La liberté du travail à Paris aux XVIre et XVIII siècles, Seyssel, Champ Vallon, 2002.

31 Luu L. B., Immigrants and the industries of London, 15001700, Ashgate, Aldershot, 2005.

32 Chirot D. et Reid A., Essential outsiders. Chinese and Jews in the modern transformation of Southern Asia and Central Europe, Seattle, University of Washington Press, 1997. $\mathrm{d}^{\prime}$ ordre territorial ${ }^{33} »$. Loin de glorifier le rôle de telle ou telle minorité, l'enjeu est de penser le fait identitaire et minoritaire hors des ethnocentrismes, hors de " l'exaltation de la grandeur d'un groupe ${ }^{34} »$, et de restituer toutes les nuances et les facettes des identités dans leurs liens aux savoirs et aux pratiques. Plusieurs pistes se présentent : comprendre les ethnocentrismes comme objets d'histoire eux-mêmes, « amarrer l'étude du local au contexte global » et privilégier ainsi l'analyse braudélienne des « recouvrements de civilisations ${ }^{35}$ », approfondir la définition des identités culturelles dans toute leur complexité, au prisme des circonstances, des conjonctures, du particulier, en somme des conditions historiques. Dans tous les cas, I'histoire des techniques joue un rôle clef tant les interrogations sur la discipline, sur son passé, sur ses mythes sont au cœur des enquêtes actuelles.

Les communications rassemblées ici en attestent. Peter Jones revient sur un thème classique de I'historiographie, les liens entre les minorités religieuses dissent dans l'Angleterre du xvIII siècle et l'essor d'une culture publique scientifique et technique, marquée par le providentialisme. Prenant l'exemple de Birmingham, célèbre dans ce domaine, I'auteur montre que si les non-conformistes, comme James Watt, appartiennent à l'élite économique de la ville, s'ils peuplent la Lunar Society, ils côtoient dans ces activités des membres de l'Église anglicane, tels Matthew Boulton. Dans la lignée de travaux de Margaret Jacob et de Larry Stewart relativisant l'impact de la culture dissent sur l'essor de la philosophie naturelle ${ }^{36}$, en mettant l'accent sur le consensus établi par le courant latitudinaire, Peter Jones souligne le rôle des partenariats entre puritains et anglicans et la communauté de vues qui assoit leur foi dans l'avancement du savoir. Les spécificités minoritaires sont revues à la

33 Denoix S., Espagne M., Miller C., Lorcerie F., « Transferts, mobilités, recompositions culturelles et politiques », Rapport de conjoncture, Paris, CNRS, 2004, pp. 211-222, p. 213.

34 Ibid., p. 214.

35 Gruzinski S., " Histoire et anthropologie, une question inactuelle? », Bulletin de la Société d'histoire moderne et contemporaine, Revue d'histoire moderne et contemporaine, vol. 49-4bis, supplément 2002, pp. 89-92.

36 Jacob M. C., The cultural meaning of the scientific revolution, New York, Alfred A. Knopf, 1988 ; Stewart, L., The rise of public science. Rhetoric, technology, and natural philosophy in Newtonian Britain, 1660-1750, Cambridge, Cambridge University Press, 1992; Jacob M. C. et Stewart L., Practical matter: Newton's science in the service of industry and empire, 1687-1851, Cambridge (Mass.), Harvard University Press, 2004. 
baisse et les mythes fondateurs sont questionnés par I'examen scrupuleux des réseaux de sociabilité.

D'autres pistes sont ouvertes par Éric Reppel pour les armuriers étrangers à Tours aux $x v^{e}$ et $x v^{e}$ siècles et par Lien Luu pour les migrants à Londres à la même période. II s'agit de comprendre ici comment les contemporains forgent les identités de groupes et de minorités. Cherchant à éviter les stéréotypes sur le rôle déterminant de telle communauté, les auteurs soulignent la fluidité des identités au travail, les migrants s'adaptant aux demandes des autorités et aux activités des communautés étrangères installées. Tous deux montrent en retour le rôle du droit et des politiques d'accueil différenciées dans l'intégration réussie ou non des étrangers, dans la reconnaissance ou le rejet de leurs compétences, dans la mise en place de représentations favorables ou xénophobes. Le propos est clair : étudier les migrants à la lumière de leur réception par les pouvoirs politiques, par les natifs et leurs compatriotes déjà sur place. Hétérogénéité des communautés, circuits complexes avec persistance des retours au pays d'origine, différenciation des modèles de diffusion selon les métiers, selon les périodes, ambiguïté des politiques migratoires et des statuts attribués : la diversité des expériences l'emporte sur tout modèle unifiant.

L'article de Giorgio Riello ajoute d'autres facettes au prisme identitaire et à sa construction par les contemporains, en fait par les passeurs eux-mêmes : si les artisans et commerçants italiens en Europe aux $x V \|^{e}$ et $x V \| I^{e}$ siècles jouent des ressources que leur procure leur origine pour valoriser des produits sur le marché, construisant à l'étranger la « distinction » italienne des produits, ces stratégies professionnelles ne recoupent pas leurs sentiments identitaires ni leurs ambitions sociales. Du moins, les cheminements sont sinueux. L'auteur montre que les stuccatori du Tessin forment une communauté « diachronique » qui au fil des générations offre ses services dans différentes villes d'Europe ; plus que le sentiment d'appartenance à une communauté, ce sont " les liens temporaires avec d'autres artisans du Tessin » qui construisent leur identité d'artisans gyrovagues, et surtout, la transmission d'une sensibilité esthétique commune entre générations de migrants. Inversement, les marchands d'aliment italiens à Londres au xvIII siècle sont résolument désireux d'intégration ; pour autant, le goût italien, culinaire et culturel, se déploie dans un public particulier, au cœur de leur système relationnel, le monde du théâtre italien. Dans les deux cas, entre la qualité des produits et l'identité des personnes n'existe aucune relation immédiate, mais des perceptions différenciées de l'appartenance à une communauté économique et culturelle.

Les travaux d'Anthony Pinto sur la reconversion des juifs et des conversos dans le textile en Catalogne à la fin du Moyen Âge permettent de préciser encore la complexité des relations entre les identités communautaires et les activités professionnelles. Concurrencés par les chrétiens dans le domaine du crédit (de plus, interdit aux nouveaux convertis), les juifs catalans se sont tournés vers le textile. À l'échelle de ce seul secteur, la réalité est multiple. Si la confection et la couture ne sont rien « le domaine privilégié des juifs» dans cette région, les judéo-convers bénéficient de l'héritage et de réseaux établis de longue date par les juifs dans l'artisanat de la soie ; de même dans le travail du lin, du chanvre et du coton. Pour autant, le phénomène reste circonscrit. Le commerce des articles textiles est plus largement investi mais dans le seul négoce des toiles de lin et de chanvre; le marché des draps est l'apanage des « vieux » chrétiens. Face à la pression économique des autorités chrétiennes, les juifs et les néophytes tentent leur chance dans des métiers où existent quelques réseaux d'appui et où les barrières d'entrée ne sont pas élevées. Loin d'exceller dans tel ou tel domaine, de se spécialiser dans une activité pérenne, de transmettre des savoir-faire aux autres, les judéo-convers font l'expérience de la ségrégation économique et à terme, de l'émigration. Leur cas rappelle que les identités professionnelles des minorités sont aussi marquées par la vie fragile, le manque de connaissances techniques et de moyens économiques, l'échec à se maintenir.

En somme, la thématique de la journée d'étude nous invite à inscrire le thème de la transmission des savoirs dans une réflexion sur les mythes des origines et sur les constructions identitaires, attribuées, revendiquées et réinventées et sur la place assignée aux techniques, à l'exercice du métier et aux choix professionnels dans I'élaboration de ces représentations. Les travaux présentés confirment que l'histoire des techniques appartient pleinement à l'histoire sociale et culturelle, non seulement en termes d'histoire des savoirs mais aussi d'imaginaires sociaux et d'idéologies. 